\title{
WIRELESS SYSTEM FOR DETERMINING TOXIC EMISSIONS FROM INDUSTRIAL ENVIRONMENTS
}

\author{
Ciprian Ion Rizescu ${ }^{1}$, Mihai Nicolae Gheorghiţă ${ }^{1}$, Dana Rizescu ${ }^{1}$ \\ 1University POLITEHNICA of Bucharest, Faculty of Mechanical Engineering and Mechatronics, \\ Department of Mechatronics and Precision Mechanics, Bucharest, Romania \\ Email: dana.rizescu@upb.ro
}

\begin{abstract}
Previously developed toxic gas detection systems typically use a wired network to transmit information, resulting in a limited monitoring area, low portability and higher investment in human resources. Fixed location detection systems cannot accurately detect the exact location of toxic gas leaks. Moreover, improving this accuracy requires more equipment, resulting in a much higher overall system cost. The proposed paper refers to the design of a wireless system for the detection of toxic gases in industrial environments. The system is based on two sensors for detecting toxic gases (MQ-7, respectively MQ-135) connected to an Arduino Uno development board. The operation of the system is quite simple, namely: when one of the sensors comes into contact with a toxic gas, a visual signal is received from a sensor-specific LED and an audio signal from the buzzer. The mechanical structure of the vehicle on which the two sensors are implemented was designed and manufactured by the authors. The work will be developed in the future by using optical sensors and spectral analysis.
\end{abstract}

Keywords: Toxic Gases Detection, Wireless System, Optic Sensors.

\section{Introduction}

As it is known poor air quality causes many deaths or illnesses each year. The lack of low-cost environmental monitoring equipment prevents people from taking steps to improve air quality. At present, environmental monitoring methods are expensive for most economic operators and offer low spatial coverage, which makes their usefulness limited.

Air quality measurements track the environmental air and detect dangerous, un-wanted gases and particulates in the air. Often these particulates and materials are invisible to the human eye, but their effect on humans and the environment can be significant.

Sensors will play a key role in IoT development and will be widely used in smart home and smart city programs. Heating, ventilation and air conditioning (HVAC) systems, air purifiers, smart windows and other applications will use sensors to improve the quality of life of people around the world. We expect a growing market for gas sensors used in smart homes and smart cities.

Air quality sensors monitor air and detect various gaseous substances, which generally contribute to air pollution or safety hazards. The chemical properties of these concentrations differ from each other, and a single sensor cannot detect all substances.
Separate sensors capable of detecting individual chemical concentrations are implemented to measure individual gas concentrations in the environment. [1]

Air contains many substances beyond its constituent portions of nitrogen and oxygen. Air quality sensors typically detect substances such as carbon dioxide (CO2), nitrogen dioxide (NO2), trioxygen (O3), carbon monoxide (CO), volatile organic compounds (VOCs) and particles. These concentrations are measured in ppm (parts per million) or ppb (parts per billion).

Previous toxic gas detection systems typically use a wired network to transmit information, resulting in a complex network launch, limited monitoring area, reduced portability, and greater investment in human resources. Fixed location detection systems cannot accurately detect the exact location of toxic gas leaks. Moreover, improving this accuracy requires more terminal equipment, which determines the overall cost of the system at a much higher level. [2]

Another important condition is the sensor calibration. Air quality sensors are calibrated for accurate working and correct readings. The continuous exposure to pollutants shifts the internal circuit function away from its standard and reference values. The sensor's outer body is continuously exposed to contaminants, which changes the sensor's material structure. 
These factors affect the sensor function, causing inconsistent readings.

Air quality sensors require calibration at regular intervals. The manufacturer provides recommendations for the calibration procedure and frequency. organizations typically must keep a record of the calibration procedure, as regulatory bodies require routine inspection activity.

The calibration can be done internally by the organization but more often, third-party experts perform this function. The regulatory bodies are certified and mandated to carry out calibration activities and provide certifications to the user.

The proposed paper refers to the design of a wireless system for the detection of toxic gases in industrial environments. The system is based on two sensors for detecting toxic gases (MQ-7, respectively MQ-135) connected to an Arduino Uno development board. The operation of the system is quite simple, namely: when one of the sensors comes into contact with a toxic gas, a visual signal is received from a sensor-specific LED and an audio signal from the buzzer.

\section{Experimental Setup}

There was developed in Inventor 2018 the design of vehicle for supporting two gas sensors: MQ-7 [3] and MQ-135 [4], as it is shown in figure 1.

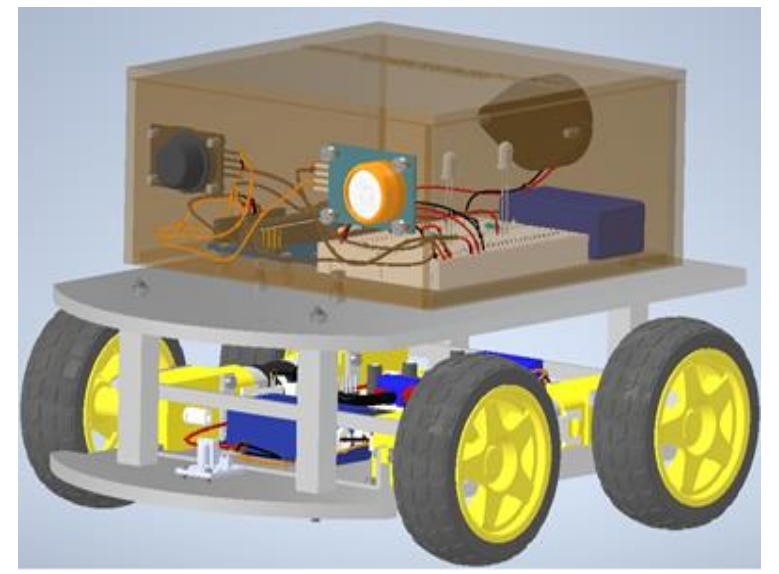

Figure 1: Designed Vehicle

MQ-7 sensors are used in gas detecting equipment for carbon monoxide (CO) in family and industry or car applications, figure 2. In Figure 3 it is presented the sensitivity characteristics of the MQ-7 sensor.

Structure and configuration of MQ-7 gas sensor is shown in figure 2 (Configuration $\mathrm{A}$ or $\mathrm{B}$ ), sensor composed by micro $\mathrm{Al}_{2} \mathrm{O}_{3}$ ceramic tube, Tin Dioxide $\left(\mathrm{SnO}_{2}\right)$ sensitive layer, measuring electrode and heater are fixed into a crust made by plastic and stainless-steel net. The heater provides necessary work conditions for work of sensitive components.
The enveloped MQ-7 have 6 pins, 4 of them are used to fetch signals, and the other 2 are used for providing heating current.
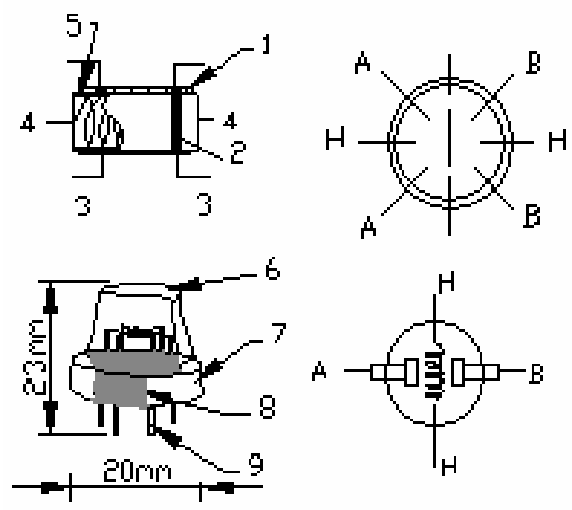

Figure 2: Gas sensor MQ-7

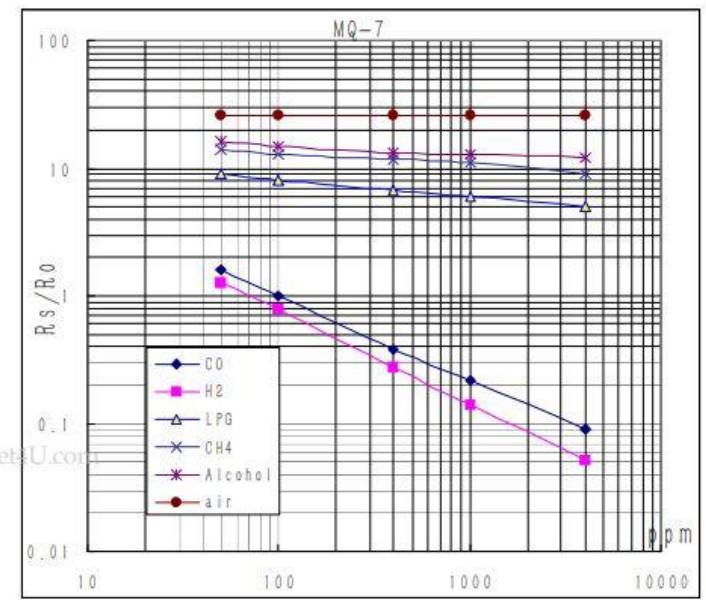

Figure 3 Sensitivity characteristics of the $M Q-7$
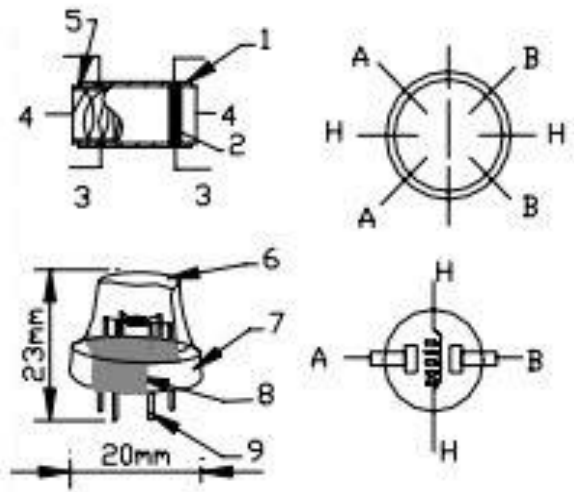

Figure 4: Gas sensor MQ-135

MQ-135 sensors are used in air quality control equipment for buildings / offices, are also suitable for detecting of $\mathrm{NH}_{3}, \mathrm{NOx}$, alcohol, Benzene, smoke, $\mathrm{CO}_{2}$, etc, figure 4 . In Figure 5 it is presented the sensitivity characteristics of the MQ-135 sensor. 
Structure and configuration of MQ-135 gas sensor is shown in figure 4 (Configuration $\mathrm{A}$ or $\mathrm{B}$ ), sensor composed by micro $\mathrm{Al}_{2} \mathrm{O}_{3}$ ceramic tube, Tin Dioxide $\left(\mathrm{SnO}_{2}\right)$ sensitive layer, measuring electrode and heater are fixed into a crust made by plastic and stainless-steel net.

The heater provides necessary work conditions for work of sensitive components. The enveloped MQ-135 have 6 pins, 4 of them are used to fetch signals, and other 2 are used for providing heating current.

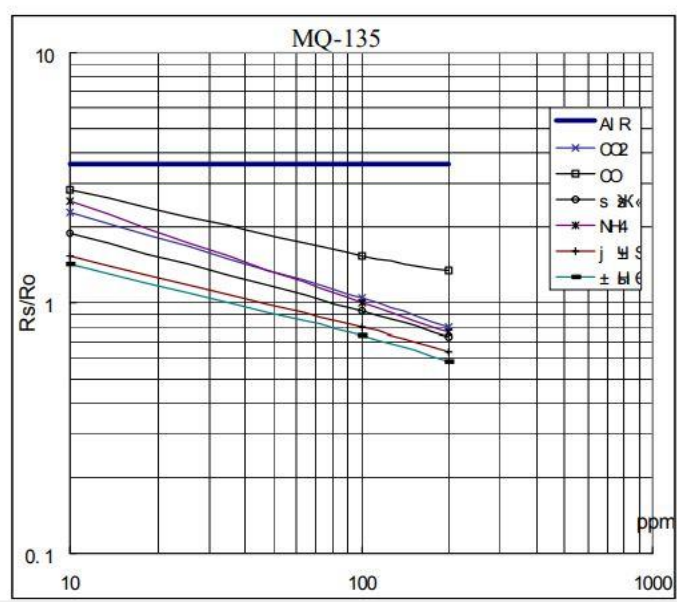

Figure 5 Sensitivity characteristics of the MQ-135

\section{The Gas Detection Simulation}

In order to design the experimental detection setup there was developed a gas detection simulation, for both sensors, using Tinkercad environment. For each sensor the experimental setup was equipped with a LED. The blue LED is connected to the MQ-7 sensor and the red Led is connected to the MQ-135 sensor.

The simulation consists of exposing the sensors to the toxic gases for which they were designed. Figure 6 shows the simulation of the MQ-7 sensor operating. When the sensor is exposed to toxic gases (carbon monoxide), an audio signal is received from the buzzer at a frequency of $5000 \mathrm{~Hz}$ and a visual signal from the blue LED. Figure 7 shows the simulation of the MQ-135 sensor operating. When the sensor is exposed to toxic gases $\left(\mathrm{NH}_{3}, \mathrm{NOx}\right.$, alcohol, benzene, smoke, $\mathrm{CO}_{2}$ ) an audio signal is received from the buzzer at a frequency of $5000 \mathrm{~Hz}$ and a visual signal from the red LED.

\section{Sensor Sensitivity Adjustment}

Figure 8 is showing the typical dependence of the MQ-7 on temperature and humidity. Ro: sensor resistance at $100 \mathrm{ppm} \mathrm{CO}$ in air at $33 \% \mathrm{RH}$ and $20^{\circ} \mathrm{C}$. Rs: sensor resistance at $100 \mathrm{ppm} \mathrm{CO}$ at different temperatures and humidity.

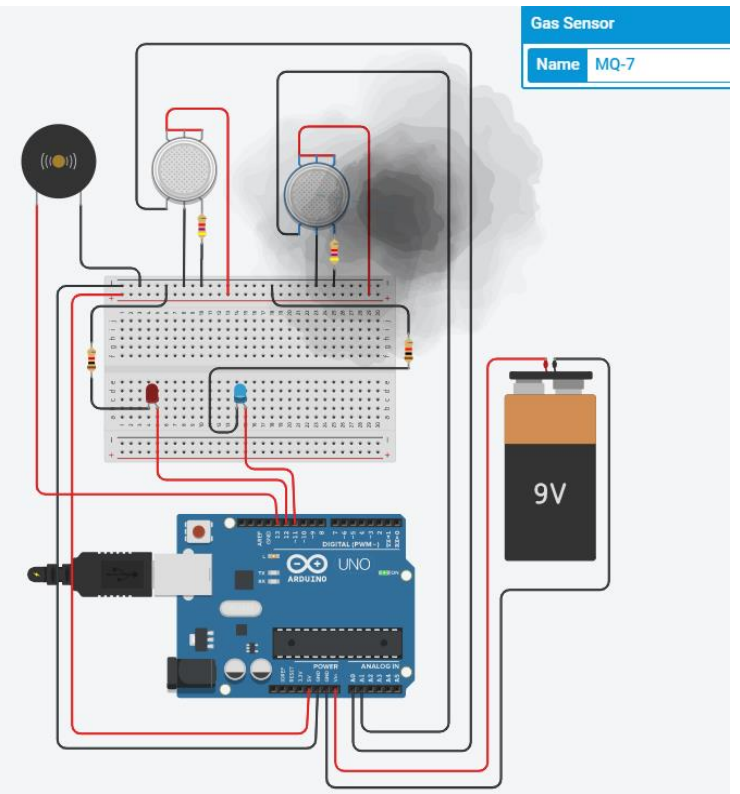

Figure 6 Simulation for sensor MQ-7 operating

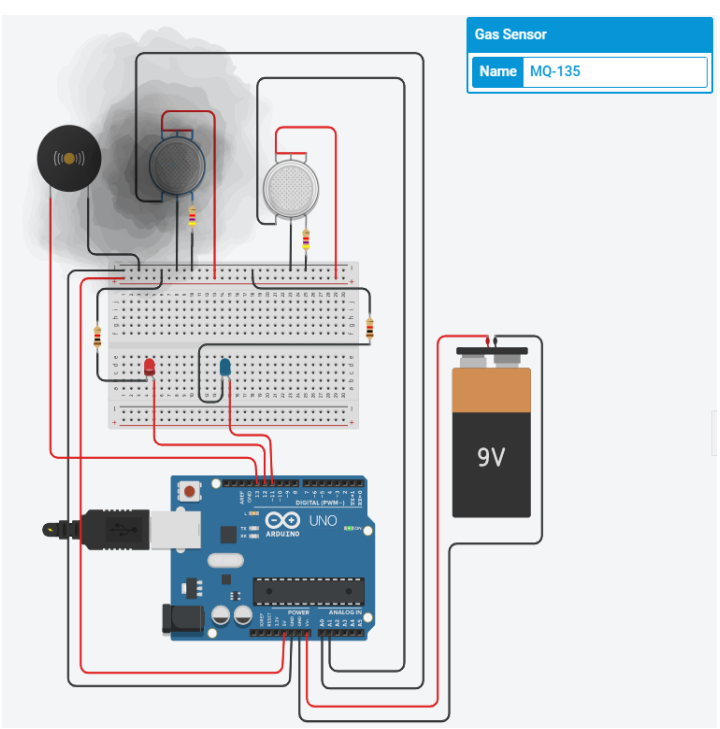

Figure 7 Simulation for sensor MQ-135 operating

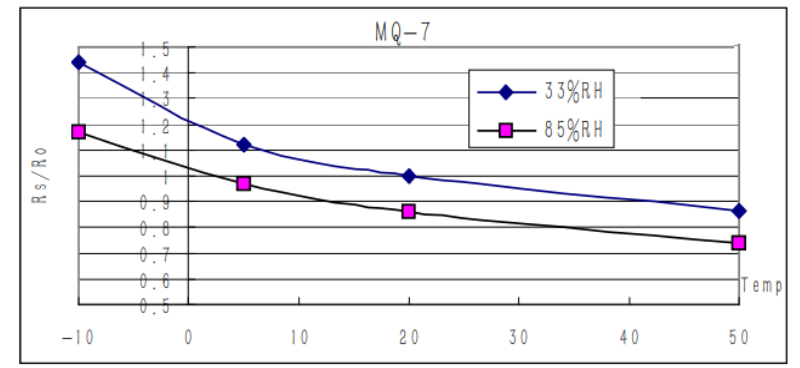

Figure 8 Resistance dependence with temperature and humidity for $M Q-7$

Resistance value of MQ-7 is difference to various kinds and various concentration gases. So, when using this component, sensitivity adjustment is very necessary. 
There is recommended to calibrate the detector for $200 \mathrm{ppm} \mathrm{CO}$ in air and use value of Load resistance that (RL) about $10 \mathrm{~K} \Omega(5 \mathrm{~K} \Omega$ to $47 \mathrm{~K} \Omega)$. When there are necessary accurately measuring operations, the proper alarm point for the gas detector should be determined after considering the temperature and humidity influence. The sensitivity adjusting program:

a. Connect the sensor to the application circuit.

b. Turn on the power, keep preheating through electricity over 48 hours.

c. Adjust the load resistance RL until you get a signal value which is respond to a certain carbon monoxide concentration at the end point of 90 seconds.

d. Adjust another load resistance RL until you get a signal value which is respond to a $\mathrm{CO}$ concentration at the end point of 60 seconds.

Figure 9 is showing the typical dependence of the MQ-135 on temperature and humidity. Ro: sensor resistance at $100 \mathrm{ppm}$ of $\mathrm{NH} 3$ in air at 33\% $\mathrm{RH}$ and $20{ }^{\circ} \mathrm{C}$. Rs: sensor resistance at $100 \mathrm{ppm}$ of $\mathrm{NH}_{3}$ at different temperatures and humidity.

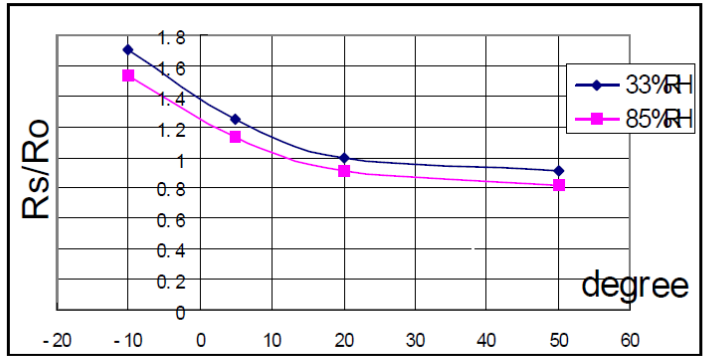

Figure 9 Resistance dependence with temperature and humidity for $M Q-135$

Resistance value of MQ-135 is difference to various kinds and various concentration gases. So, when using these components, sensitivity adjustment is very necessary. There is recommended to calibrate the detector for $100 \mathrm{ppm} \mathrm{NH3}$ or $50 \mathrm{ppm}$ Alcohol concentration in air and use value of Load resistance that (RL) about $20 \mathrm{~K} \Omega(10 \mathrm{~K} \Omega$ to $47 \mathrm{~K} \Omega)$. When it is necessary accurately measuring work, the proper alarm point for the gas detector should be determined after considering the temperature and humidity influence.

\section{Vehicle for Gas Detection}

The authors designed a system to transport the gas sensors. The system consists of a chassis equipped with four c.c. motors, each motor actuating a driving wheel. In figure 10 it is presented the block diagram of the actuation and control system. Concerning the actuation of the four motors, one of the easiest and inexpensive way to control stepper motors is to interface L293D Motor Driver IC with Arduino [5].
It can control both speed and spinning direction of any Unipolar stepper motor.

Arduino UNO is a microcontroller board based on the ATmega328P. It has 14 digital input/output pins (of which 6 can be used as PWM outputs), 6 analogue inputs, a $16 \mathrm{MHz}$ ceramic resonator, a USB connection, a power jack, an ICSP header and a reset button. It contains everything needed to support the microcontroller; simply connect it to a computer with a USB cable or power it with a AC-to-DC adapter or battery to get started. The main components of the actuation and control system are presented in figure 11 .

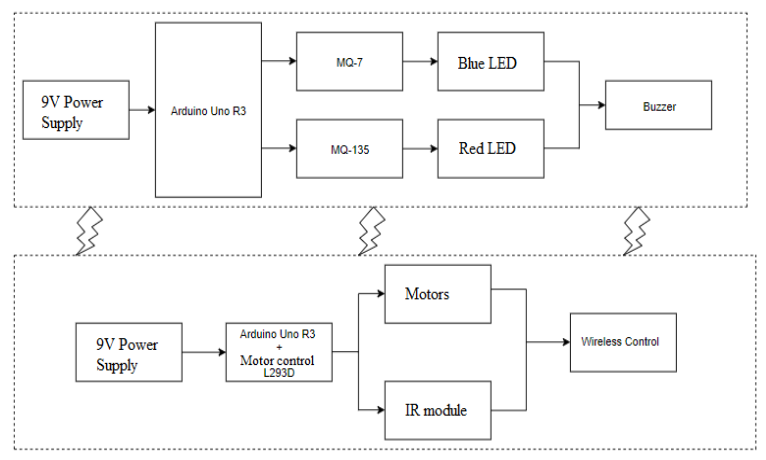

Figure 10The block diagram of actuation and control system

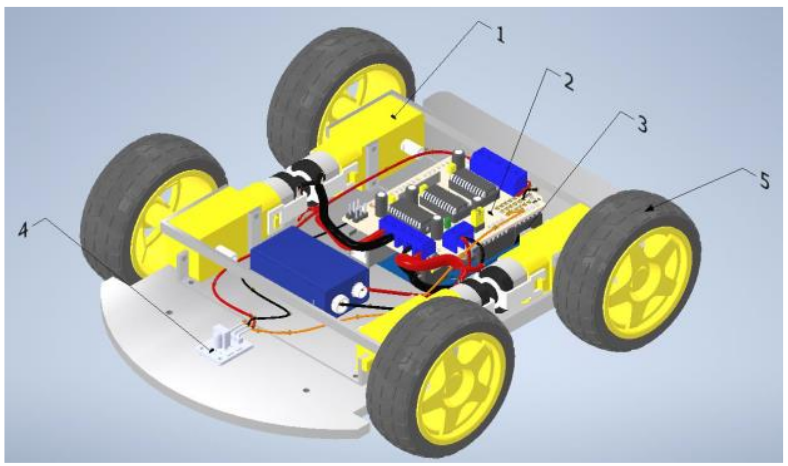

Figure 11 Main components of the actuation and control system

The main components of the experimental setup figured in figure 1 are:

1. C.C. motor with speed reducer;

2. L293D chip for motors control;

3. Arduino UNO R3.

4. KY-022 infrared receiver module [6];

5. Vehicle wheel.

This module, KY-022 infrared module, consists of a 1838 IR receiver, a $1 \mathrm{k} \Omega$ resistor and a LED. It works together with the KY-005 IR transmitter module. Compatible with popular electronic platforms like: Arduino [7], Raspberry $\mathrm{Pi}$ and ESP8266. Arduino IR receiver module KY-022, reacts to $38 \mathrm{kHz}$ infrared light.

In figure 12 is shown the assembly between electrical motor and speed reducer. The motor speed is 9624 [rpm] and the speed ratio is 35.5 . 


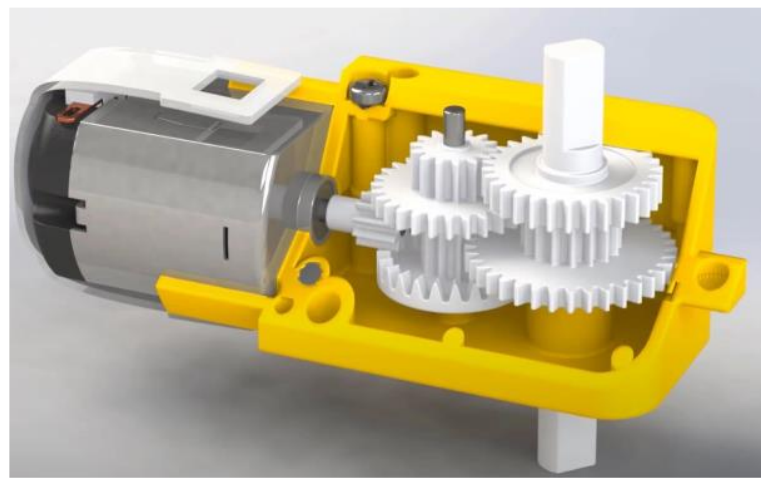

Figure 12.CC. Motor and speed reducer

To detect toxic gas leaks, it is proposed to expose the sensors to 3 types of toxic gases. The following tests were performed:

1.Sample 1 consists of the smoke resulting from the burning of a piece of wood, figure 13.

2.Sample 2 consists of the leakage of liquefied gas from a cylinder, figure 14 .

3. Sample 3 consists of isopropyl alcohol.

The humidity inside, shown for experimental tests - samples 1 and 2 in figures 13,14 is $45.5 \%$ and the temperature is $27.7^{\circ} \mathrm{C}$, both determined with the Data Logger DT-171 device, connected to PC via USB port, [8]. All experimental tests were developed in indoor spaces. For outdoor spaces the detecting system must have some improvements. For open spaces, disturbing factors such as wind direction and wind speed, distance from the source of toxic gases must be considered. In this case, determining the humidity and temperature is a challenge.
The results of the measurements can be significantly affected in the case of open spaces.

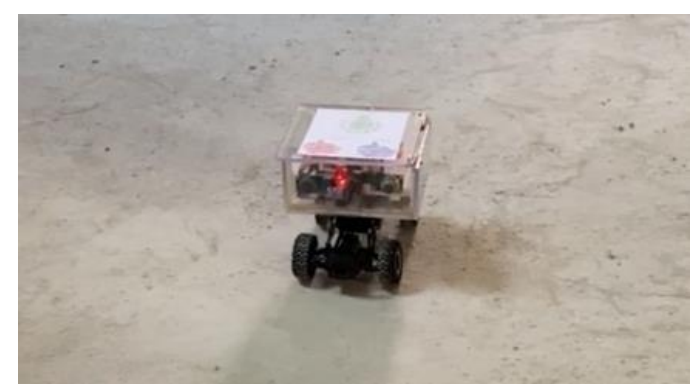

Figure 13 Experimental setup detecting smoke

The results of temperature and humidity determination for Sample 1 and sample 2 are presented in figure 15. For all three determinations the detection time was less than $10 \mathrm{~s}$, the reaction of the sensors was very fast. In the future we will study the effect of aging sensors but also the possibility of using an optical sensor to detect microparticles $[9,10]$.

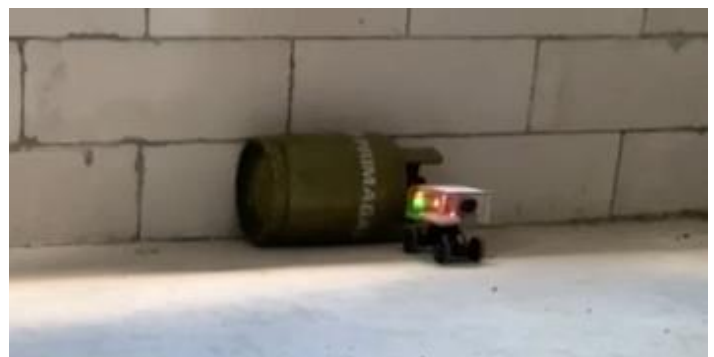

Figure 14 Experimental setup detecting gas leaking

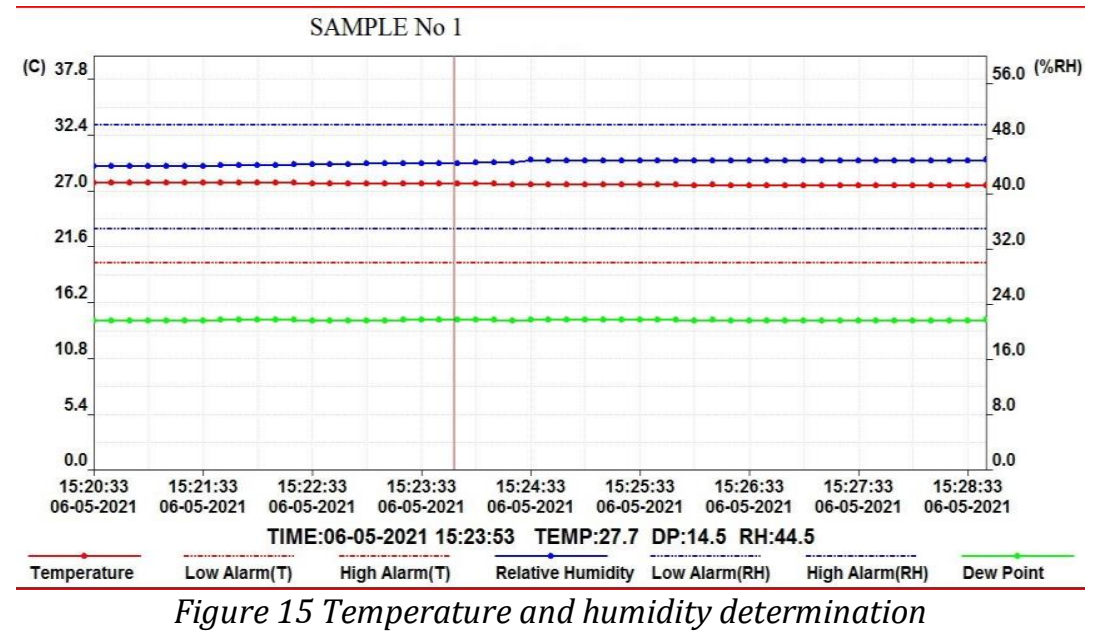

Sensors that detect particulate matter (PM) use optical sensors. PM consists of solid and liquid particles [11]. When these particles are inhaled by humans, serious health problems could result.

These sensors work by measuring the amount of light scattered by the particles as the sample air passes through a beam of light. The light is scattered due to the concentration of particulates in the sample air, and the detector's algorithm converts the scattering pattern into particulate size and amount.
The reading of these sensors depends upon several physical particle parameters such as shape, colour, and density.

The optical sensing technology is also used for different gases such as $\mathrm{CO}$ and $\mathrm{CO}_{2}$. The authors intend to implement these optical sensors in the future in certain applications such as the detection of $\mathrm{CO}$ and $\mathrm{CO}_{2}$ emissions $[12,13]$. There is also the problem of unknown toxic gas emissions, in which the choice of sensor is decisive $[14,15]$. 


\section{Conclusions}

The objective of the developed work was to design a wireless system that aims to detect several toxic gases in industrial environments. Gas is an important place in everyone's life, and its widespread use will inevitably lead to problems with toxic gas leaks. In view of the above, in order to keep the staff of companies in this field safe, the existence of a wireless detection device is significant.

Following the analysis, we notice that this system, which is based on two sensors connected to the Arduino Uno development board and which works thanks to a fairly simple system, has as its main purpose the improvement of the quality of life of people around the world.

Improving previous existing systems brings high costs. Older devices that use a wired network to transmit information limit the monitoring area and bring reduced portability and greater investment in human resources. Instead, wireless detection systems have a direct process by which one of the sensors that comes in contact with a toxic gas will transmit both a visual and an auditory signal.

These types of sensors will play an important and significant role in many areas and in many areas of development, helping to improve the quality of human existence, both in terms of health and economics.

The authors believe that this system will bring significant benefits and therefore aim to further develop research, wanting to bring and find as many improvements as possible, such as:

- Implementation of a video camera for the visibility of remote system operation;

- Implementation of a device on the system to transmit data about the type of gas detected, its concentration and level of toxicity;

- Implementation of optical sensor that detect particulate matter;

- Replacement of current sensors with sensors that have a more detailed accuracy (detection of gases with a higher degree of toxicity than that encountered regularly). Replacement of current sensors with sensors that have a more detailed accuracy (detection of gases with a higher degree of toxicity than that encountered regularly).

In the future, to satisfy additional several customer needs, the authors would like to develop smaller sensors that can be incorporated into any of the items described above, as well as sensors equipped with a dust concentration output function that can give more accurate information to users.
Our future applications should cover the subsequent areas: City atmospheric environmental monitoring, Enterprise environment monitoring, Factory area unorganized emission monitoring, Environment evaluation monitoring, Portable gas detector, Various gas detection equipment and smart home appliance.

\section{References}

[1] „M. A. Nlazi, „Insights global spec,” 09 June 2021. [Interactive]. Available: https://insights.globalspec.com/article/16765/f undamentals-of-air-quality-sensors. accessed at:29.10.2021

[2] K.E. Kakosimos. Marc J. Assael, „Fires, Explosions, and Toxic Gas Dispersions, Effects Calculation and Risk Analysis" CRC Press Taylor \& Francis Group 2010.

[3] https://datasheetspdf.com/pdf/694312/Hanwei /MQ7/1 accessed at: 29.10.2021

[4] https://datasheetspdf.com/pdf/605077/Hanwei /MQ135/1 accessed at: 29.10.2021

[5] https://www.instructables.com/Arduino-Howto-Control-a-Stepper-Motor-With-L293D-/

[6] https://www.epitran.it/ebayDrive/datasheet/45. pdf accessed at: 29.10.2021

[7] https://www.farnell.com/datasheets/1682209.p df, accessed at: 29.10.2021

[8] https://www.ceminstruments.in/product.php?pname=DT-171, accessed at: 29.10.2021

[9] https://www.gas-sensing.com/aeroqual-pmsensor.html, accessed at: 29.10.2021

[10] https://www.alphasense.com/products/optical -particle-counter/, accessed at:29.10.2021

[11] https://www.alphasense.com/wpcontent/uploads/2021/05/OPC-R2.pdf, accessed at: 29.10 .2021

[12] https://www.winsensensor.com/sensors/toxic-gassensor/ze12.html?campaignid=10464432613\&a dgroupid=107390153001\&feeditemid=\&targetid =kwd-

$420767116065 \&$ device $=c \&$ creative $=446277584$ 598\&keyword=optical\%20gas\%20sensor\&gclid= CjwKCAjw2vOLBhBPEiwAjEeK9v09EyhJoYolcS5 ExCm1X_Nld4uPZ7teiRxEXLSj3P7A8vRaw1y7xoCrtUQAvD_BwE accessed at: 30.10.2021

[13] https://dekatitechnologies.com/particlemeasurement-solutions/pm-sensors/ at: 30.10.2021

[14] https://ipo.llnl.gov/technologies/instrumentssensors-and-electronics/optical-sensorunknown-gas-detection accessed at: 30.10.2021

[15] https://www.techbriefs.com/component/conte nt/article/tb/supplements/st/briefs/27504 accessed at: 30.10 .2021 